has no discernible inhibitory effect on $\beta$ cell expansion. Taken together, these findings cast doubt on the likelihood that insulin or Igf1 play more than a possible permissive role in the $\beta$ cell expansion of insulin resistance. Perhaps what have been called the insulin signaling pathways in $\beta$ cells should be viewed as growth and survival pathways used by glucose, cAMP, and a variety of growth factors. Although we have focused on the obviously important Irs $2 / \mathrm{PI} 3 \mathrm{~K} / \mathrm{PKB}$ pathway, the complete picture of the signals responsible for $\beta$ cell growth is no doubt far more complex.

The fact that this tidy reduction of Gck in $\beta$ cells prevents $\beta$ cell expansion in an excellent model of insulin resistance (5) adds great strength to the hypothesis that glucose is the dominant force responsible for this important compensatory mechanism. But, as with any hypothesis, future rigorous challenge is awaited. Nonetheless, because of the importance of $\beta$ cell failure in $\mathrm{T} 2 \mathrm{D}$ and the power of new therapeutic approaches, it is essential that glucose signals involved in $\beta$ cell replication in both health and disease be carefully defined.

\section{Acknowledgments}

The authors thank Christopher J. Rhodes for helpful discussion and advice.
Address correspondence to: Gordon C. Weir, Section on Islet Transplantation and Cell Biology, Joslin Diabetes Center, One Joslin Place, Boston, Massachusetts 02215, USA. Phone: (617) 732-2581; Fax: (617) 732-2650; E-mail: gordon.weir@ joslin.harvard.edu.

1. Bruning, J.C., et al. 1997. Development of a novel polygenic model of NIDDM in mice heterozygous for IR and IRS-1 null alleles. Cell. 88:561-572.

2. Butler, A.E., et al. 2003. Beta-cell deficit and increased beta-cell apoptosis in humans with type 2 diabetes. Diabetes. 52:102-110.

3. Ritzel, R.A., Butler, A.E., Rizza, R.A., Veldhuis, J.D., and Butler, P.C. 2006. Relationship between betacell mass and fasting blood glucose concentration in humans. Diabetes Care. 29:717-718.

4. Bonner-Weir, S. 2000. Perspective: postnatal pancreatic beta cell growth. Endocrinology. 141:1926-1929.

5. Terauchi, Y., et al. 2007. Glucokinase and IRS-2 are required for compensatory $\beta$ cell hyperplasia in response to high-fat diet-induced insulin resistance. J. Clin. Invest. 117:246-257. doi:10.1172/ JCI17645.

6. Matschinsky, F.M. 1996. A lesson in metabolic regulation inspired by the glucokinase glucose sensor paradigm. Diabetes. 45:223-241.

7. Chen, C., Hosokawa, H., Bumbalo, L.M., and Leahy, J.L. 1994. Regulatory effects of glucose on the catalytic activity and cellular content of glucokinase in the pancreatic B cell. J. Clin. Invest. 94:1616-1620.

8. Hosokawa, H., Hosokawa, Y.A., and Leahy, J.L. 1995. Upregulated hexokinase activity in isolated islets from diabetic $90 \%$ pancreatectomized rats. Diabetes. 44:1328-1333.

9. Kim, S.P., Catalano, K.J., Hsu, I.R., Chiu, J.D., and Bergman, R. 2004. Elevated nocturnal FFA may be the signal for compensatory hyperinsulinemia for insulin resistance induced by a high fat diet in the dog model [abstract]. Obes. Res. 11(Suppl. 1):A14.

10. Withers, D.J., et al. 1998. Disruption of IRS-2 causes type 2 diabetes in mice. Nature. 391:900-904.

11. Rhodes, C.J. 2005. Type 2 diabetes-a matter of betacell life and death? Science. 307:380-384.

12. Park, S., et al. 2006. Exendin-4 uses Irs 2 signaling to mediate pancreatic beta cell growth and function. J. Biol. Chem. 281:1159-1168.

13. Jhala, U.S., et al. 2003. cAMP promotes pancreatic beta-cell survival via CREB-mediated induction of IRS2. Genes Dev. 17:1575-1580.

14. Okamoto, H., et al. 2006. Role of the forkhead protein FoxO1 in $\beta$ cell compensation to insulin resistance. J. Clin. Invest. 116:775-782. doi:10.1172/JCI24967.

15. Lingohr, M.K., et al. 2006. Specific regulation of IRS-2 expression by glucose in rat primary pancreatic islet beta-cells. J. Biol. Chem. 281:15884-15892.

16. Kulkarni, R.N., et al. 1999. Tissue-specific knockout of the insulin receptor in pancreatic b cells creates an insulin secretory defect similar to that in type 2 diabetes. Cell. 96:329-339.

17. Kulkarni, R.N., et al. 2002. beta-cell-specific deletion of the Igf1 receptor leads to hyperinsulinemia and glucose intolerance but does not alter beta-cell mass. Nat. Genet. 31:111-115.

18. Ueki, K., et al. 2006. Total insulin and IGF-I resistance in pancreatic beta cells causes overt diabetes. Nat. Genet. 38:583-588.

19. Duvillie, B., et al. 1997. Phenotypic alterations in insulin-deficient mutant mice. Proc. Natl. Acad. Sci. U. S. A. 94:5137-5140.

20. Laybutt, D.R., et al. 2002. Overexpression of c-Myc in beta-cells of transgenic mice causes proliferation and apoptosis, downregulation of insulin gene expression, and diabetes. Diabetes. 51:1793-1804.

21. Miyaura, C., et al. 1991. Expression of reg/PSP, a pancreatic exocrine gene: relationship to changes in islet beta-cell mass. Mol. Endocrinol. 5:226-234.

\title{
Prostaglandin-secreting cells: a portable first aid kit for tissue repair
}

\author{
Seth Rakoff-Nahoum and Ruslan Medzhitov
}

Howard Hughes Medical Institute (HHMI) and Section of Immunobiology, Yale University School of Medicine, New Haven, Connecticut, USA.

\begin{abstract}
After intestinal injury, both the number and type of intestinal epithelial cells must be restored. Intestinal stem cells, located at the base of the intestinal crypt, repopulate the depleted crypt in a process known as compensatory proliferation. In this issue of the JCI, Brown et al. describe a new mechanism by which this process is regulated (see the related article beginning on page 258). Surprisingly, they find that a subset of stromal cells present within the intestinal tissue and expressing the proliferative factor prostaglandinendoperoxidase synthase 2 (Ptgs2) is repositioned next to the intestinal stem cell compartment where local production of $\mathrm{PGE}_{2}$ controls injury-induced epithelial cell proliferation.
\end{abstract}

Nonstandard abbreviations used: DSS, dextran sodium sulfate; PSC, prostaglandin-expressing stromal cell; Ptgs2, prostaglandin-endoperoxide synthase 2 .

Conflict of interest: The authors have declared that no conflict of interest exists.

Citation for this article: J. Clin. Invest. 117:83-86 (2007). doi:10.1172/JCI30865.
Most tissues of complex metazoans are renewable, which provides an obvious advantage for animals with an extended life span. In some tissues (for example, skin and intestinal epithelium), newly differentiated cells simply replace damaged and worn-out older cells. In the immune system, on the other hand, continuous replacement of lymphocytes is needed to maintain the diversity of antigen receptor specificities. The cell turnover rates vary widely among different tissues, with hematopoietic and intestinal epithelial cells, at the extreme end of the spectrum, characterized by the highest rate of renewal of as quick as 2-3 days. In all cases, tissue renewal is afforded by the presence of adult stem cells, which can produce new terminally differentiated cells in the steady state and as part of a tissue repair response triggered by injury-induced cell loss. The proliferative state of stem cells must therefore be regulated by extrinsic cues that report the tissue status and thus adjust the rate of tissue renewal. The nature of these signals, how they are regulated, and whether 

A Normal rectal

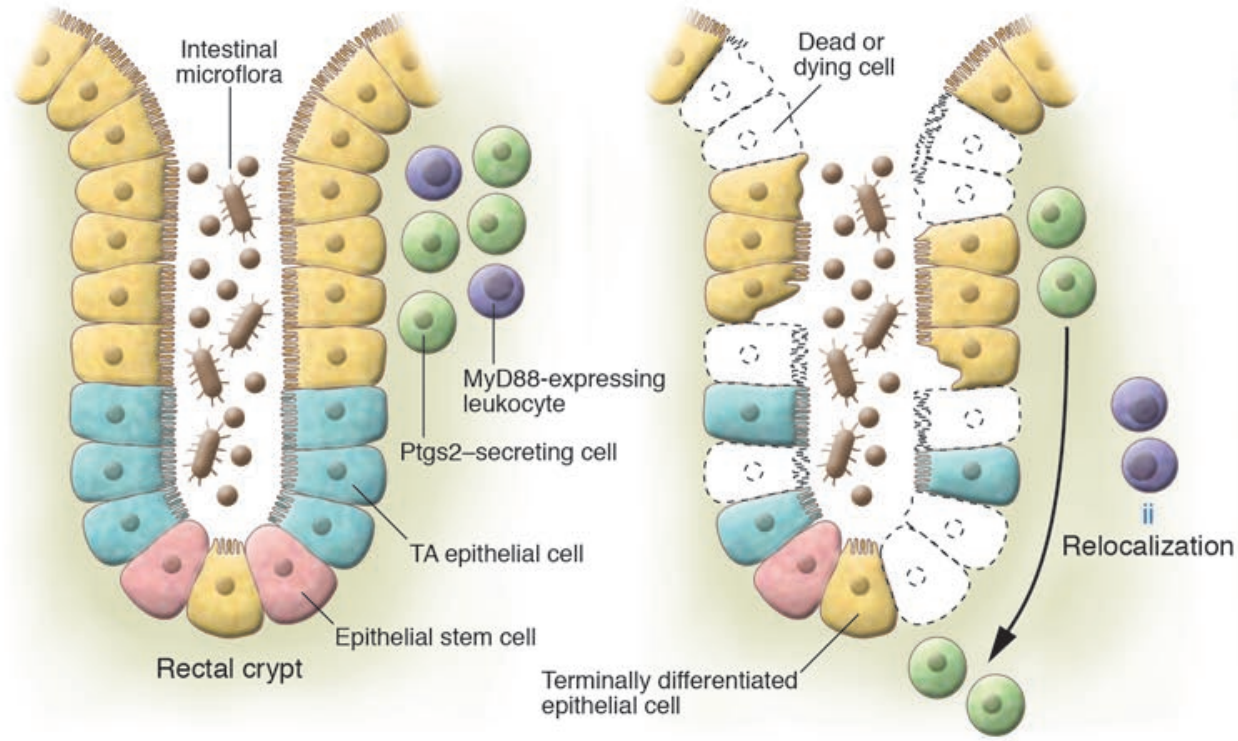

C

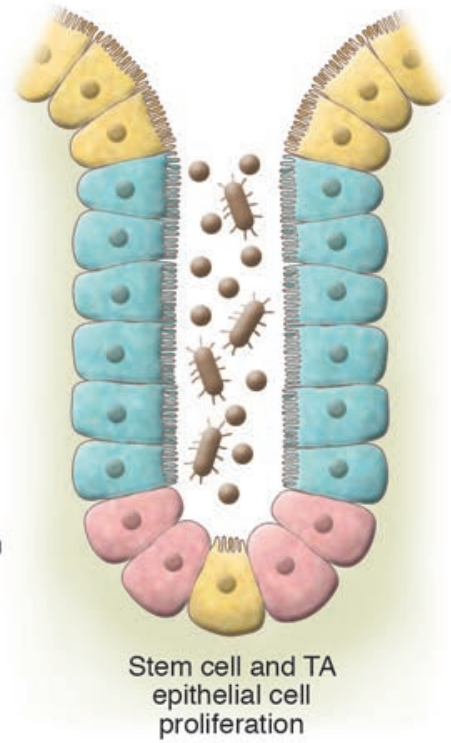

Figure 1

Model of MyD88-dependent relocalization of Ptgs2-expressing cells to the rectal crypt base and epithelial proliferation following DSS-induced injury. (A) In the steady state, Ptgs2-expressing epithelial cells are mostly present in the lamina propria of the upper and middle regions of the rectal crypt. (B) Upon DSS-induced injury (i), these Ptgs2-expressing cells migrate to the bottom of the crypt, occupying a position near the stem cell niche (ii). This relocalization is dependent on MyD88 expression by leukocytes, presumably stimulated by TLR recognition of microbial products following barrier disruption. (C) Compensatory proliferation of stem cells and transit-amplifying (TA) epithelial cells after DSS-induced

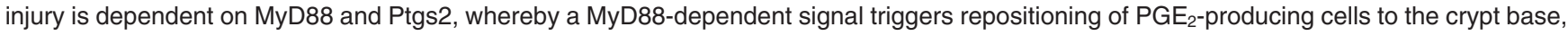
adjacent to the stem cell compartment.

they act directly on stem cells or indirectly through the niche cells (cells neighboring stem cells and regulating their functions), is unknown in most cases. However, there is an enormous interest in answering these questions given their importance for understanding normal tissue homeostasis, tissue repair, and tumorigenesis.

\section{The intestine as a model of tissue renewal and regeneration}

The mammalian intestine is arguably the best model system with which to study the regulation of steady-state tissue renewal and the injury-induced tissue repair response. The small and large intestines of mammals are composed of cells from each of the 3 embryonic layers. The microarchitecture of the intestine consists of cryptvillous units (in the small intestine) and elongated crypts (in the colon). These units are lined with epithelial cells. The tops of the crypts and villi contain terminally differentiated cells, such as enteroabsorptive, goblet, and neuroendocrine cells. All of the differentiated intestinal epithelial cells arise from multipotent precursors, known as transit-amplifying cells, located closer to the bottom of the crypt. Hidden at the crypt base are the intestinal epithelial stem cells (Figure 1A).

In addition to epithelial cells, the intestinal tissue contains cells of mesenchymal origin, including leukocytes and fibroblasts. These cells are arranged in various locations throughout the crypts and villi, with the majority present in the lamina propria compartment of the mucosa below the epithelial basement membrane.

In the steady state, there is a modest amount of epithelial turnover in the intestine (1). At any given moment, there are a few epithelial cells dying at the top of the crypts. Physiologic cell loss is balanced by low-level, constant division of cells originating from the totipotent stem cells at the base of the crypt.

The proliferation of intestinal epithelial progenitor cells is also adaptable to circumstances under which there is a need to renew more than the steady-state number of cells. The intestinal epithelium plays an important role as a functional barrier and must be able to withstand widespread cell death due to ingestion of toxic compounds, microbial infection, inflammation, mechanical stress- es, and iatrogenic causes including chemotherapy and radiation therapy.

Although tissue renewal and homeostasis are probably best understood for intestinal epithelium, many fundamental questions remain. How do intestinal epithelial cells know when to proliferate, i.e., how is injury perceived? Do these stimuli or signals of injury act directly on progenitor cells by converging on pathways known to regulate their proliferation (e.g., Wnt, bone morphogenic protein, Hedgehog, or Notch signaling pathways [ref. 2])? Or, alternatively, is injury perceived by mesenchymal cells, which in turn instruct progenitor cells to proliferate? The findings reported by Brown et al. (3) in this issue of the JCI add a surprising new dimension to these fundamental questions and suggest what we believe to be a novel mechanism for sensing tissue injury and initiating the tissue repair response in the colon.

\section{Innate immune recognition of the commensal microflora as a cue for intestinal tissue repair}

The lumen of mammalian intestine is home to trillions of microbes, predominantly bacteria, that encompass the indigenous com- 
mensal microflora (4). Recent studies with conventionally raised mice depleted of commensal bacteria $(5)$ or germ-free mice $(6,7)$ administered the colonic epithelial toxin dextran sodium sulfate (DSS) have led to the conclusion that the microflora are required for protection from DSS-induced colonic injury and for induction of compensatory proliferation. Commensal bacteria contribute to tissue protection and repair by stimulating TLRs, which are best known for their essential functions in pathogen detection and protection from infection (8). Mice deficient in TLR2 and TLR4 (which recognize conserved bacterial products such as lipopeptides and LPS) or the TLR signaling adaptor myeloid differentiation factor 88 (MyD88) are phenotypically similar to germfree mice with regard to protection from intestinal injury and subsequent compensatory proliferation $(5,7,9,10)$. Thus perception of injury and induction of tissue repair processes may occur via recognition of commensal microbial products that gain access to the TLRs expressed on myeloid cells upon epithelial barrier disruption $(7,11)$.

How then does TLR recognition of commensal bacteria lead to the induction of epithelial proliferation following injury? A number of studies implicated various growth factors in mediating epithelial regeneration after injury (12). Of these, $\mathrm{PGE}_{2}$, a biologically active and pleiotropic eicosanoid, has been shown to be particularly important for protection from colonic injury. $\mathrm{PGE}_{2}$ is a product of prostaglandin-endoperoxide synthase 2 (Ptgs2, also known as COX-2). Ptgs2-deficient mice show more colonic injury than WT controls upon DSS administration (13), and intraperitoneal injection of $\mathrm{PGE}_{2}$ increases epithelial proliferation after DSS exposure in WT mice (14). The TLR/MyD88 signaling pathway positively regulates Ptgs2 $(15,16)$, and in one study, isolated mesenchyme from DSS-treated descending colons revealed a MyD88-dependent induction of Ptgs 2 mRNA (7). Based on these prior findings, it was reasonable to hypothesize that Ptgs2 may play a critical role in TLR/MyD88-dependent protection from epithelial injury in the colon.

In their studies, Brown, Stappenbeck, and colleagues (3) focused on the rectum, a region of the colon that is spared the severe injury that occurs upon DSS administration at the descending colon. They found that the rectal tissue of both $M y d 88^{-/-}$and Ptgs2 $2^{-/}$mice has similar defects in rectal crypt architecture and epithelial proliferation following injury compared with that of WT mice. The authors provide evidence that both MyD88 and Ptgs 2 were part of the same signaling pathway, as administration of exogenous $\mathrm{PGE}_{2}$ to $M y d 88^{-/-}$mice rescued the defect in epithelial compensatory proliferation after injury.

Having established a functional relationship between MyD88 and Ptgs2 in the control of epithelial proliferation, Brown et al. (3) went on to investigate the mechanism of Ptgs 2 regulation. It is here that things got intriguing. In contrast to what one would expect, the authors found that although rectal mesenchyme expresses Ptgs 2 mRNA during the steady state, DSS administration did not increase Ptgs 2 mRNA levels, though it did induce the expression of other genes. Interestingly, they identified what is believed to be a novel cell type in the rectal epithelial tissue that expresses Ptgs2 at high levels at the steady state and which the authors refer to as prostaglandin-expressing stromal cells (PSCs). Importantly, rectal injury did not lead to an increase in the number of PSCs or an increase in the rectal secretion of $\mathrm{PGE}_{2}$. Instead, DSS-induced epithelial injury led to a repositioning of the PSCs.

In the steady state, the majority of PSCs were located in the lamina propria lining the upper and middle third of the rectal crypt (Figure 1A). However, upon DSS-induced injury, the number of PSCs at these locales decreased and the number of PSCs increased at the crypt base, adjacent to the intestinal stem compartment (Figure 1B). The injuryinduced relocalization of Ptgs2-expressing

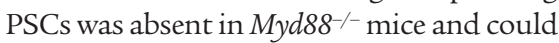
be rescued by adoptive transfer of peripheral blood leukocytes from Rag1 1/- (T cell- and B cell-deficient) mice, suggesting that MyD88 signaling in a cell derived from a nonlymphoid hematopoietic lineage, most likely the macrophage, is involved in the in situ repositioning of PSCs upon injury.

This observation of MyD88-dependent relocalization of PSCs to the crypt base (3), a region designated the "stem cell niche," suggests that the local production of PGE $_{2}$ by PSCs may be the mechanism by which MyD88 and Ptgs 2 are linked in regulating rectal epithelial proliferation after injury. It remains to be determined whether the $\mathrm{PGE}_{2}$ produced by these relocated PSCs at the regenerating crypt base acts directly on epithelial progenitor cells to induce their proliferation (a $\mathrm{PGE}_{2}$ receptor, EP2, is expressed on regenerating epithelium in the small intestine [ref. 17]); acts on other cells, such as enteric neurons, that can trigger progenitor cell proliferation
(18); or mediates an ancillary process such as angiogenesis (19) that may be required to support epithelial proliferation mediated by MyD88- or Ptgs2-independent pathways.

Further studies will undoubtedly reveal how MyD88 signaling in leukocytes leads to the movement of PSCs from the upper regions to the base of rectal crypts. TLR/ MyD88 signaling induces many chemokines in addition to tissue remodeling factors such as MMPs. Thus, recruitment by chemoattraction and modulation of the extracellular matrix may be involved in the relocalization of PSCs during rectal injury. Identifying these MyD88-dependent processes will be an exciting avenue for future research. It would also be important to investigate whether the mechanism described by Brown et al. (3) operates in other parts of the colon and the small intestine.

TLR/MyD88 signaling is responsible for both transcriptional regulation of Ptgs $2(7,20)$ and increased Ptgs 2 protein expression by macrophages (20). In the proximal colon, Ptgs2 seems to play a primary role in negatively regulating the acute infiltration of leukocytes upon DSS-induced injury (13). Furthermore, while $\mathrm{PGE}_{2}$ seems to be important in small intestinal crypt survival and proliferation following radiation injury, this is due to COX-1 rather than Ptgs 2 activity (21). It would be interesting to find out why different modes of regulation of $\mathrm{PGE}_{2}$ production have evolved and what aspects of the intestinal tissue physiology determine the choice of regulatory mechanism. It would also be worth examining whether a similar mechanism, based on repositioning of signal-emitting cells, is involved in the regulation of $\mathrm{PGE}_{2}$ production in other physiological and developmental settings. Like any other discovery, the findings by Brown et al. (3) point to many new exciting possibilities.

Address correspondence to: Ruslan Medzhitov, HHMI and Section of Immunobiology, Yale University School of Medicine, 300 Cedar Street, New Haven, Connecticut 06405, USA. Phone: (203) 785-7541; Fax: (203)785-4461; E-mail: ruslan.medzhitov@ yale.edu.

\footnotetext{
1. Marshman, E., Booth, C., and Potten, C.S. 2002. The intestinal epithelial stem cell. Bioessays. 24:91-98.

2. Radtke, F., and Clevers, H. 2005. Self-renewal and cancer of the gut: two sides of a coin. Science. 307:1904-1909.

3. Brown, S.L., et al. 2007. Myd88-dependent positioning of Ptgs2-expressing stromal cells maintains colonic epithelial proliferation during injury. J. Clin. Invest. 117:258-269. doi:10.1172/JCI29159.

4. Ley, R.E., Peterson, D.A., and Gordon, J.I. 2006. Ecological and evolutionary forces shaping microbial
} 
diversity in the human intestine. Cell. 124:837-848. 5. Rakoff-Nahoum, S., Paglino, J., Eslami-Varzaneh, F., Edberg, S., and Medzhitov, R. 2004. Recognition of commensal microflora by toll-like receptors is required for intestinal homeostasis. Cell. 118:229-241

6. Kitajima, S., Morimoto, M., Sagara, E., Shimizu, C., and Ikeda, Y. 2001. Dextran sodium sulfateinduced colitis in germ-free IQI/Jic mice. Exp. Anim. 50:387-395

7. Pull, S.L., Doherty, J.M., Mills, J.C., Gordon, J.I., and Stappenbeck, T.S. 2005. Activated macrophages are an adaptive element of the colonic epithelial progenitor niche necessary for regenerative responses to injury. Proc. Natl. Acad. Sci. U. S. A. 102:99-104.

8. Takeda, K., Kaisho, T., and Akira, S. 2003. Toll-like receptors. Annu. Rev. Immunol. 21:335-376.

9. Araki, A., et al. 2005. MyD88-deficient mice develop severe intestinal inflammation in dextran sodium sulfate colitis. J. Gastroenterol. 40:16-23.

10. Fukata, M., et al. 2005. Toll-like receptor-4 is required for intestinal response to epithelial injury and limiting bacterial translocation in a murine model of acute colitis. Am. J. Physiol. Gastrointest. Liver Physiol. 288:G1055-G1065.

11. Rakoff-Nahoum, S., Hao, L., and Medzhitov, R. 2006. Role of toll-like receptors in spontaneous commensaldependent colitis. Immunity. 25:319-329.

12. Booth, D., and Potten, C.S. 2001. Protection against mucosal injury by growth factors and cytokines. J. Natl. Cancer Inst. Monogr. 2001:16-20.

13. Morteau, O., et al. 2000. Impaired mucosal defense to acute colonic injury in mice lacking cyclooxygenase-1 or cyclooxygenase-2.J. Clin. Invest. 105:469-478.

14. Tessner, T.G., Cohn, S.M., Schloemann, S., and Stenson, W.F. 1998. Prostaglandins prevent decreased epithelial cell proliferation associated with dextran sodium sulfate injury in mice. Gastroenterology. 115:874-882.

15. Rhee, S.H., and Hwang, D. 2000. Murine TOLLlike receptor 4 confers lipopolysaccharide responsiveness as determined by activation of NF kappa $\mathrm{B}$ and expression of the inducible cyclooxygenase. J. Biol. Chem. 275:34035-34040.

16. Eliopoulos, A.G., Dumitru, C.D., Wang, C.C., Cho,
J., and Tsichlis, P.N. 2002. Induction of COX-2 by LPS in macrophages is regulated by Tpl2-dependent CREB activation signals. EMBO J. 21:4831-4840.

17. Houchen, C.W., Sturmoski, M.A., Anant, S., Breyer, R.M., and Stenson, W.F. 2003. Prosurvival and antiapoptotic effects of PGE2 in radiation injury are mediated by EP2 receptor in intestine. Am. J. Physiol. Gastrointest. Liver Physiol. 284:G490-G498.

18. Bjerknes, M., and Cheng, H. 2001. Modulation of specific intestinal epithelial progenitors by enteric neurons. Proc. Natl. Acad. Sci. U. S. A. 98:12497-12502.

19. Wang, D., et al. 2006. CXCL1 induced by prostaglandin E2 promotes angiogenesis in colorectal cancer. J. Exp. Med. 203:941-951.

20. Fukata, M., et al. 2006. Cox-2 is regulated by Tolllike receptor-4 (TLR4) signaling: role in proliferation and apoptosis in the intestine. Gastroenterology. 131:862-877.

21. Houchen, C.W., Stenson, W.F., and Cohn, S.M. 2000. Disruption of cyclooxygenase-1 gene results in an impaired response to radiation injury. Am. J. Physiol. Gastrointest. Liver Physiol. 279:G858-G865.

\title{
Beta blocker specificity: a building block toward personalized medicine
}

\author{
Brent R. DeGeorge Jr. and Walter J. Koch \\ Center for Translational Medicine and George Zallie and Family Laboratory for Cardiovascular Gene Therapy, \\ Department of Medicine, Thomas Jefferson University, Philadelphia, Pennsylvania, USA.
}

\begin{abstract}
Drugs known as beta blockers, which antagonize the $\beta$-adrenergic receptor $(\beta-A R)$, are an important component of the treatment regimen for chronic heart failure (HF). However, a significant body of evidence indicates that genetic heterogeneity at the level of the $\beta_{1}$-AR may be a factor in explaining the variable responses of HF patients to beta blockade. In this issue of the JCI, Rochais et al. describe how a single amino acid change in $\beta_{1}$-AR alters its structural conformation and improves its functional response to carvedilol, a beta blocker currently used in the treatment of HF (see the related article beginning on page 229). This may explain why some HF patients have better responses not only to carvedilol but to certain other beta blockers as well. The data greatly enhance our mechanistic understanding of myocardial adrenergic signaling and support the development of "tailored" or "personalized" medicine, in which specific therapies could be prescribed based on a patient's genotype.
\end{abstract}

Chronic heart failure (HF) poses a major public health problem in this country, primarily due to the increasing proportion of our society surviving to the seventh and eighth decades of life and to improved treatment of acute ischemic cardiac events, resulting in more survi-

Nonstandard abbreviations used: AC, adenylyl cyclase; $\beta$-AR, $\beta$-adrenergic receptor; FRET, fluorescence resonance energy transfer; GPCR, G protein-coupled receptor; $\mathrm{HF}$, heart failure.

Conflict of interest: The authors have declared that no conflict of interest exists.

Citation for this article: J. Clin. Invest. 117:86-89 (2007). doi:10.1172/JCI30476. vors who develop cardiac dysfunction. The group of drugs known as beta blockers block the effects of catecholamines, such as epinephrine and norepinephrine, on the body's $\beta$-adrenergic receptors ( $\beta$-ARs), slowing nerve impulses traveling through the heart and reducing the heart's workload. While $\beta$-AR antagonists have become a mainstay of HF therapy, the most recent guidelines from the American College of Cardiology and the American Heart Association acknowledge that there are unresolved issues concerning the use of these drugs for the treatment of HF (1).

\section{$\beta_{1}$-AR polymorphisms and cardiac phenotype}

The $\beta$-ARs are members of the $G$ proteincoupled receptor (GPCR) superfamily, which consists of over 700 genes that are the targets of more than $50 \%$ of the drugs in clinical practice (2). There are 3 known types of $\beta$-ARs, $\beta_{1}, \beta_{2}$, and $\beta_{3}$. The $\beta_{1}$-ARs are located mainly in the heart, kidney, and adipose tissue. The $\beta_{2}$-ARs are located mainly in the heart, lung, gastrointestinal tract, liver, pancreas, and skeletal muscle. The role and location of $\beta_{3}$-ARs are less well defined. When stimulated by agonists, $\beta$-ARs primarily activate heterotrimeric guanine nucleotide-binding $(G)$ proteins of the Gs family, causing dissociation of G $\alpha-G T P$ and $\mathrm{G} \beta \gamma$ subunits. The $\mathrm{G}$ proteins transduce intracellular signaling pathways via adenylyl cyclase (AC) activation, and this results in increased intracellular cAMP levels (3). This signaling cascade ultimately leads to positive regulatory input to the myocardial contractile apparatus. Importantly, $\beta$-ARs present on cardiomyocytes represent the most powerful means of enhancing the contractile performance of the heart.

The predominant $\beta$-AR subtypes of functional consequence to cardiac physiology are $\beta_{1}-\mathrm{AR}$ and $\beta_{2}-\mathrm{AR}$, both of which have 